\title{
Simultaneous selection on vegetative and reproductive phenology in a perennial herb
}

\author{
Elsa Fogelström ${ }^{1}$, Giulia Zacchello ${ }^{2}$, and Johan Ehrlen ${ }^{1}$ \\ ${ }^{1}$ Stockholm University \\ ${ }^{2}$ Uppsala Universitet
}

October 6, 2021

\begin{abstract}
The timing of different life history events are often correlated, and selection might only rarely be exerted independently on the timing of a single event. In plants, phenotypic selection has often been shown to favour earlier flowering. However, little is known about to what extent this selection acts directly vs. indirectly via vegetative phenology, and if selection on the two traits is correlational. We estimated direct, indirect and correlational phenotypic selection on vegetative and reproductive phenology over three years for the perennial herb Lathyrus vernus. Direct selection favoured earlier flowering and shorter timespans between leaf-out and flowering in all years. However, early flowering was associated with early leaf-out, and the direction of selection on leaf-out day varied among years. As a result, selection on leaf-out weakened selection for early flowering in one of the study years. We found no evidence of correlational selection. Our results highlight the importance of including temporally correlated traits when exploring selection on the phenology of seasonal events.
\end{abstract}

\section{Introduction}

In seasonal environments, timing of events such as emergence, growth, reproduction and seasonal senescence is often temporally and developmentally correlated (Rathcke and Lacey 1985, Kelly 1992, O'Neil 1997, Aizen 2003, Sola and Ehrlén 2007, Keenan and Richardson 2015). Such correlations imply that selection might only rarely be exerted independently on the timing of a single event. Instead, selection on a focal phenological trait is often a combination of direct selection, and indirect selection acting via other phenological traits (Rathcke and Lacey 1985, Kelly 1992, Ehrlén 2015, Galloway et al. 2018). Selection could also target the relative timing of phenological traits. Such selection has been found for development time to maturity and reproduction in both plants and animals (Kingsolver and Pfennig 2004). Lastly, the strength or direction of selection on a focal trait might often depend on the level of other phenological traits (correlational selection; Lande and Arnold 1983, Phillips and Arnold 1989, Kelly 1992). Taken together, this implies that to accurately estimate selection acting on a focal phenological trait, it is necessary to account for indirect selection via temporally correlated traits, as well as for correlational selection.

In temperate plants, the timing of reproduction is a particularly important life-history trait, as it influences interactions with the biotic and abiotic environment and is often strongly linked to fitness (e.g. Elzinga et al. 2007, Ehrlén 2015, Austen et al. 2017). Phenotypic selection has been found to favour early flowering in many temperate plant species (Harder and Johnson 2009, Munguía-Rosas et al. 2011), and the consistency of this pattern has raised questions as to why plants do not appear to respond to this selection (Austen et al. 2017). One suggested explanation for the observed pattern is that selection on flowering time is exerted indirectly via correlated life-history traits and that this indirect selection is not accounted for in analyses (cf. Rathcke and Lacey 1985, Austen et al. 2017). For example, several studies have documented correlations 
between flowering time and vegetative phenology (e.g. Kelly 1992, Diggle 1999, Yao and Mehlenbacher 2000, Sola and Ehrlén 2007, Brachi et al. 2012). If vegetative and reproductive spring phenology are correlated, evolution of flowering time is influenced also by selection on timing of vegetative phenology, and selection will act simultaneously on both traits. In these cases, we expect direct and indirect selection on both traits, as well as on their relationship. Yet, very few studies have simultaneously examined selection on vegetative and reproductive phenology, and quantified direct, indirect and correlational selection (but see Kelly 1992).

In this study, we explored the relationship between vegetative spring phenology and flowering phenology in a natural population of the perennial understorey herb Lathyrus vernus in Sweden, and used multiple regressions to estimate phenotypic selection on these two traits. Previous studies with this system have found that selection favours earlier flowering in most years (Ehrlén and Münzbergová 2009, Ehrlén and Valdés 2020), but also that flowering time is correlated with start of shoot growth and leaf development (Sola and Ehrlén 2007). It is thus possible that some of the observed selection on flowering time is exerted indirectly, via selection on vegetative phenology. We expected that most of the among-year differences in the distributions of phenological traits in $L$. vernus are driven by climatic conditions (Ehrlén and Valdés 2020), while within-year differences among individuals in these traits are caused by other factors, including genetic differences. We also expected the causes of selection to both depend on the density and frequency of phenological trait values within years (soft selection, e.g. in terms of among-individual competition for pollinators, and avoidance of seed-predation and vertebrate grazing), and to be density-independent (hard selection, e.g. in terms of effects of weather conditions) (cf. De Lisle and Svensson 2017). To assess selection on vegetative and reproductive spring phenology, we monitored leaf-out day and first flowering day, and recorded individual fitness in terms of seed production, during three years. We addressed four specific questions: 1) How closely are vegetative and reproductive phenology correlated? 2) Is there phenotypic selection acting on vegetative and reproductive phenology? 3) If so, to what extent is selection on vegetative and reproductive phenology direct vs. indirect? 4) Is there selection on the relationship between vegetative and reproductive phenology, in terms of selection for time of development between leaf-out and flowering or correlational selection?

\section{Materials and methods}

\section{Study system}

Lathyrus vernus (L. Bernh) is a perennial herb that is distributed over Europe and North-West Asia (POWO 2019). In the study area, L. vernus is mainly found in the understorey of deciduous or mixed-deciduous forests. Each plant individual produces one to several shoots that emerge early in spring and that can reach up to $40 \mathrm{~cm}$ in height (Ehrlén 2002). The leaves are pinnate and consist of 2-4 pairs of leaflets that unfold starting with the basalmost leaf on the shoot and the pair of leaflets closest to the stem. Each flowering individual produces 1 to 5 racemes, each having 1 to 9 flowers with the basalmost flowers opening first (Ehrlén 2002). The racemes emerge from leaf axes and start development when the neighbouring leaf unfolds. Racemes and their adjacent leaf develop simultaneously, and although flowers normally open after that leaflets have unfolded, the first flower might sometimes open before the first leaflet has unfolded. A previous study with this species showed that plants with an earlier leaf development initiate flowering earlier than plants with a later leaf development (Sola and Ehrlén 2007). In South-East Sweden, where this study was carried out, flowering usually starts in late April to early May. Fruits contain up to 18 ovules, and the mature seeds are dispersed ballistically about 2 months after flowering (Ehrlén 1992). L. vernus reproduces only sexually.

The reproductive performance of $L$. vernus is influenced by several abiotic and biotic factors, some of which have been linked to selection on flowering time. Cold April temperatures are associated with weaker selection for early flowering, possibly because frost damage reduces the benefit of flowering early (Ehrlén and Valdés 2020). Grazing by roe deer (Capreolus capreolus ) favours later flowering because early-flowering 
individuals experience the highest levels of damage (Fogelström and Ehrlén 2019). L. vernus individuals rely on pollinators (Bombus sp.) for their reproduction and seed production is sometimes pollen limited (Ehrlén 1992), suggesting that there are fitness benefits of synchronising flowering with a high availability of bumblebees. Bruchus atomarius (Chrysomelidae) larvae can damage a large proportion of the seeds before dispersal in the study area. B. atomarius seed predation is sometimes linked to the timing of flowering in L. vernus, but the relationship is generally weak and its direction varies among years (Ehrlén 1996, Ehrlén and Münzbergová 2009).

\section{Data collection}

This study was conducted in a mixed deciduous forest at Kålsö, Sweden $\left(58^{\circ} 56^{\prime} \mathrm{N}, 17^{\circ} 39^{\prime} \mathrm{E}\right)$, in the years 2013 to 2015. We recorded all individuals in the population from shoots emergence in spring each year. We only included individuals that had not been damaged (e.g. by trampling, falling tree branches or by molluscs) before the first recording. Just before the first leaves in the population started to unfold, we increased the frequency of recordings to once or twice per week and continued recordings until both leaf-unfolding and flowering were terminated. During the study, individuals started to leaf-out 23 April - 20 May 2013, 2 April - 14 May 2014, and 26 March - 5 May 2015. Individuals initiated flowering 10 - 28 May in 2013, 18 April - 25 May in 2014, and 20 April - 23 May in 2015, respectively. The average time between leaf-out and first flowering day was 10 days in 2013, 18 days in 2014 and 20 days in 2015.

We used the estimated day of year when the first leaflet unfolded as a measure of vegetative spring phenology. Leaflets open sequentially in L. vernus, and we recorded the number of unfolded leaflets at each visit. Each individual was assigned a leaf-out day within the interval between the recording when the first leaflet was observed to be unfolded and the recording prior. We then used the proportion of open leaflets at the first recording to estimate the most probable first day with any open leaflets within that interval. We assumed that individuals with a larger proportion of open leaflets had started to leaf out earlier than individuals with a smaller proportion, and used a linear model including the total number of leaflets and its squared term to predict the number of unfolded leaflets at the first recording (Appendix S1, Table S1). Based on the deviation of the observed proportion of unfolded leaflets from this predicted proportion, we assigned individuals to a most likely leaf-out day within each recording interval, i.e. plants with larger deviations from the predicted proportion of unfolded leaflets were assigned earlier leaf-out dates than individuals with lower values (Appendix S1, Figure S1). We assigned a leaf-out day to all individuals that were undamaged at leaf-out and that had been recorded on at least one occasion before leaf-out.

We used the day of year when the first flower opened (first flowering day) as a measure of flowering phenology. We recorded the size of the largest bud for each flowering individual at each visit up to flowering. The size of the largest bud at the recording before the recording of the first open flower was then used to assign a first flowering day value when first flowering day occurred between two recordings (Appendix S2). First flowering day for individuals grazed before the first flower was observed was estimated using information about the relationship between first flowering day and bud size, day of year for the bud size observation, and aboveground volume of intact individuals (Appendix S2). We used the number of days from of leaf-out to first flowering day as a measure of relative timing. We refer to this timespan as "development time" henceforward.

The total number of shoots and the height and diameter of the focal shoot was measured in early July in each year. We then calculated the size of each individual in terms of the aboveground volume, using the formula for the volume of a cylinder, applying this to a focal shoot, and multiplying the resulting volume with the number of shoots (aboveground volume $=(0.5 \times \text { shoot diameter })^{2} \times$ shoot height $\times \pi \times$ number of shoots) During each visit in spring, we recorded incidences of roe-deer grazing. The final height of individuals grazed before the last recording in spring was estimated from the shoot height-diameter relationship of non-grazed plants (Appendix S3). For individuals that had been grazed between the last recording in spring and the final shoot recording in early July, we used the maximum recorded height in spring as an estimate of final shoot height. 
We used the number of developed seeds that had not been damaged by $B$. atomarius larvae, as a measure of plant fitness. We collected all fruits in the population in early July each year. The number of seeds in fruits that had not yet opened was counted in the field, and each seed was checked for B. atomarius entry holes. The number of seeds in fruits that had opened prior to collection in the field was estimated at the lab at Stockholm University. We counted the total number of seeds from clearly visible indentations made by the seeds in the pod walls. We also registered the number of B. atomariusentry holes in the pod wall, and estimated the proportion of seeds that had been preyed upon from the relationship between the proportions of seeds preyed upon and the number of $B$. atomarius entrance holes in the pod wall of intact fruits. We used the relationship between these two variables established in a previous study with $L$. vernus to estimate seed predation (proportion of seeds preyed upon $=1-\mathrm{e}^{-1.218}$ B. atomarius entry holes per seed; Supporting Information Appendix S3 in Fogelström and Ehrlén 2019). We were able to get estimates of size, vegetative phenology, reproductive phenology and fitness from 198, 207 and 207 individuals in 2013, 2014 and 2015, respectively.

\section{Statistical analyses}

All statistical analyses were carried out in R version 4.0.5 (R Core Team 2021). Analyses were carried out separately for each year because a large proportion of individuals (46.1\%) were recorded in only one year, and including individual as a random effect in mixed effects models could render the models unstable (Harrison et al. 2018). Summary statistics for the variables used in the analyses are presented in Appendix S4.

To estimate the strength of the correlation between leaf-out day and first flowering day, we calculated Pearson's $r$ for the untransformed variables We estimated phenotypic selection on leaf-out and first flowering day using linear models (Lande and Arnold 1983). Before analysis, we standardized leaf-out day, first flowering day, and the development time between leaf-out and first flowering day $((x-x) / \sigma)$ to a mean of 0 and unit standard deviation. Aboveground volume was transformed to its natural logarithm before standardization. Fitness was relativized $(x / x)$ to a mean of 1 . For soft selection, we expect the selection surface to be related to the flowering time of a focal individual relative to other individuals within a given year, rather than to day number. For hard selection, it is possible that the selective surface is similar across years, e.g. in terms of the probability of cold weather conditions, but that individuals experience different parts of this surface in different years. To examine selection corresponding to these two scenarios, we ran two sets of selection models that were based on relativizing fitness and standardizing traits within and across years, respectively (De Lisle and Svensson 2017). These two sets of models yielded similar results, and we present the results based on local within-year relativizations and standardizations in the main text, and the results for global across years relativizations and standardizations in Appendix S5. A relatively large proportion of the flowering individuals produced no seeds (67\% in 2013, 31\% in 2014 and $48 \%$ in 2015), and thus the residuals from the linear models did not meet the assumption of normality. We therefore evaluated the significance for the estimates from all linear models by estimating $95 \%$ bias-corrected and accelerated (BCa) bootstrap intervals using the function 'Boot' from the package 'car' version 3.0-10 with 10000 replications (Fox and Weisberg 2019 ) and 'boot.ci' in the package 'boot' version 1.3-27 (Davison and Hinkley 1997, Canty and Ripley 2021) in R. Estimates for which the $95 \%$ BCa interval does not overlap 0 are considered significant at $\alpha=0.05$.

To estimate total selection on leaf-out and first flowering day, we estimated linear selection differentials from simple linear regression models for the two traits, with fitness as a response variable and the trait as the predictor variable. We estimated nonlinear selection by adding a squared term to each model. To reduce bias of the selection estimates caused by differences plant condition, we included plant size as a covariate in these regression models (cf. Rausher 1992). Below, we report the selection estimates from these models including size as "total selection". The results of corresponding models without size are presented in Appendix S6.

To investigate to what extent selection on first flowering day was direct vs. indirect via leaf-out day, we estimated direct selection in terms of linear selection gradients for leaf-out and first flowering day from a multiple linear regression with fitness as a response variable, and aboveground volume as a covariate. We then examined whether these estimates of direct selection differed from the estimates of total selection on leaf-out and first flowering day. We considered estimates to differ if the estimate of direct selection was 
outside the BCa intervals for the estimate of total selection on each trait. Differences between the estimate of direct selection and the estimate of total selection on leaf-out day or first flowering day were interpreted as that selection was exerted indirectly, via the other phenological trait.

We investigated selection on the relationship between leaf-out and first flowering day in two ways: First, we estimated selection on the development time from leaf-out to first flowering day, using linear models with relative fitness as a response variable, development time as a predictor variable, and plant size as a covariate. Second, we estimated correlational selection by including the interaction leaf-out day $\times$ first flowering day in the multiple linear regression model with relative fitness as a response variable, leaf-out and first flowering day as predictor variables and with plant size as a covariate (cf. Brodie 1992). A statistically supported effect of the leaf-out day $\times$ first flowering day interaction indicates that there is correlational selection on the two traits, i.e. that the strength or direction of selection on flowering time and timing of leaf-out is dependent on the level of the other trait. Selection on development time, on the other hand, could occur regardless of whether leaf-out and flowering time are correlated or not, and regardless of whether selection on flowering time is dependent on the timing of leaf-out or not.

Lastly, we ran models with leaf-out day ${ }^{2}$ and first flowering day ${ }^{2}$ added to the model estimating correlational selection, to estimate the nonlinear selection gradients. All nonlinear selection estimates were doubled to obtain more accurate measures of the magnitude of nonlinear selection (Stinchcombe et al. 2008).

\section{Results}

Leaf-out and first flowering day were significantly positively correlated in all years, and correlation coefficients ranged from 0.32 to 0.46 (Pearson's correlation; 2013: $\mathrm{r}=0.46, \mathrm{t}_{196}=7.29, \mathrm{p}<0.001 ; 2014: \mathrm{r}=0.41, \mathrm{t}_{201}$ $=6.44, \mathrm{p}<0.001 ; 2015: \mathrm{r}=0.32, \mathrm{t}_{205}=4.77, \mathrm{p}<0.001 ;$ Fig. 1$)$.

There was total selection for earlier leaf-out in all years (Table 1a). Selection on leaf-out day in 2015 was nonlinear, fitness being highest in individuals with an early to intermediate leaf-out day (Table 1a; Fig. 2a). There was total selection for earlier flowering in 2013 and 2014, but not in 2015 (Table 1b; Fig. 2b). We found no evidence of nonlinear selection on first flowering day in any of the study years (Table 1b).

There was direct selection for earlier leaf-out in 2013, but in 2015 selection favoured later leaf-out instead (Table 1c; Fig. 2a). There was no direct selection on leaf-out in 2014. Direct phenotypic selection favoured earlier flowering in all three years (Table 1c; Fig. 2b). We found no support for nonlinear selection on leaf-out or first flowering day in any of the study years.

The estimate of direct selection on leaf-out in 2014 was just outside the BCa interval for the corresponding estimate of total selection, suggesting that selection for earlier leaf-out 2014 acted largely via first flowering day (Table 1a,c). The estimates of total and direct selection on leaf-out day in 2013 and 2015, and on first flowering day in all years, did not differ significantly (Table 1b; Fig. 2).

Individuals with shorter development time between leaf-out and first flowering day had higher fitness than individuals with longer development time in 2014 and 2015, but not in 2013 (Appendix S7, Table S1; Fig. 2c). We found no evidence of correlational selection on leaf-out and first flowering day in any of the three study years (Table 1c).

\section{Discussion}

For understorey plants in temperate regions, leafing out and flowering before canopy closure should be important for fitness (Ida and Kudo 2008, Augspurger 2008). Because the timing of these two events are often developmentally and temporally correlated, selection on either event cannot be accurately estimated independently (Lande and Arnold 1983, Kelly 1992, Diggle 1999). We showed that early leaf-out was consistently 
associated with early flowering in a population of L. vernus, and that phenotypic selection was acting on both traits. The direction of direct selection on leaf-out day differed among years, whereas direct selection consistently favoured earlier flowering. Selection on leaf-out day acted indirectly, via flowering time, in one of the three years. Selection favoured shorter development times in two of three years. There was no evidence for correlational selection on leaf-out and first flowering day. Taken together, our results show that flowering phenology is correlated with vegetative phenology during spring, and that selection on vegetative phenology can affect selection on flowering time. Still, in our study selection favoured early onset of flowering in all three years study, also when accounting for indirect selection via leaf-out day.

Leaf-out and first flowering day were significantly positively correlated in all three years, implying that flowering time is constrained by the timing of leaf-out in $L$. vernus, i.e. individuals must start their vegetative development early in order to flower early (cf. Diggle 1999, Sola and Ehrlén 2007). At the same time, there was also considerable independent variation in the two traits. Some of this variation might be attributed to differences in shoot architecture, in terms of the placement of the first inflorescence on the shoot relative to the first leaf (Diggle 1999, Sola and Ehrlén 2007). The moderately strong association between timing leaf-out and flowering initiation found in this study is in accordance with the results for other herb species (e.g. Kelly 1992, Dahlgren et al. 2007), and suggests that selection can act independently on each trait, as well as on relative timing.

We found direct phenotypic selection on leaf-out day in two of three years. Interestingly, selection was in opposite directions in these two years. Such among-year differences in the direction of selection might be related changes in trait means due to plastic responses to inter-annual variation in spring temperature. In our study, selection favoured earlier leaf-out in the year when development in spring was on average latest, but favoured later leaf-out in the year when average spring development was fastest. This pattern could be the result of that early leaf-out, relative to the population mean, implies a larger risk in years when temperatures during early spring are higher and development on average starts earlier, and that the benefits of an early development are larger in years when development on average starts later. Little is known about the agents of selection on leaf-out time in plants, but for plants where shoot development starts in early spring it is likely that weather conditions, e.g. in terms of the timing of snowmelt or late frosts events, constitute important agents of selection (cf. Inouye 2008, Augspurger 2013). In the alpine shrub Salix herbacea, selection favoured intermediate leaf-out time in sites with late snowmelt, and early leaf-out in sites with early snowmelt (Sedlacek et al. 2015), suggesting that variation in the direction of selection was mediated by the local climate. Late spring frosts likely often mediate selection for later spring development, as suggested by a study reporting that late frost events in spring primarily damaged plants in later developmental stages (Augspurger 2013). Selection on leaf-out could also be mediated by seasonal variation in light availability, and individuals that leaf-out early in spring before canopy closure are likely to have a fitness advantage due to a longer period of resource acquisition. Leafing out before canopy closure has been shown to be important for the growth and survival of understorey tree saplings (Augspurger 2008). Early spring phenology might also infer costs in terms of increased herbivory (Roy et al. 2004, Sedlacek et al. 2015). In L. vernus, grazing is likely to be the most intense early in the season when there are fewer alternative food sources, and it is possible that also variation in grazing intensity among years contributed to the observed variation in selection on vegetative phenology in our study ( $7 \%$ of individuals were grazed in $2013,26 \%$ in 2014 and $38 \%$ in 2015).

The consistent selection for early flowering found in this study is in accordance with the results of previous studies with L. vernus, as well as with several other species (Harder and Johnson 2009, Munguía-Rosas et al. 2011, Ehrlén and Valdés 2020). In our study, this pattern persisted also after taking variation in vegetative phenology into account. In addition, the results were similar for analyses that relativized fitness and standardized phenology traits within vs. across years (Table 1, Appendix S5). Selection for early flowering in L. vernus has been found to be mediated by warm April temperatures (Ehrlén and Valdés 2020). In many understorey species, early flowering is likely advantageous because light availability. and possibly pollinator activity, decrease rapidly as the canopy develops (Bertin and Sholes 1993, Ida and Kudo 2008, McKinney and Goodell 2010). This advantage is likely to be particularly large under warm spring conditions. Taken 
together, selection on both leaf-out and first flowering day in L. vernus is likely driven by multiple biotic and abiotic factors that vary in intensity and relative importance among years.

Although it has been suggested that selection on flowering time can be mediated by correlated life-history traits, for example via correlations between flowering initiation and flowering duration or between flowering time and emergence time (Rathcke and Lacey 1985, Austen et al. 2017), we are not aware of any previous study simultaneously estimating indirect selection on flowering time and leaf-out day. In our study, there was indirect selection for early leaf-out via start of flowering in 2014, suggesting that early flowering initiation was a main benefit of early leaf-out in that year. We found no statistical support for indirect selection on flowering time acting via leaf-out day, and overall our results suggest that observed consistent selection for early flowering in L. vernus is not driven by indirect selection via the timing of leaf-out. Still, selection for later leaf-out might to some extent have counteracted and weakened selection for early flowering in one of our study years; selection for early flowering in 2015 was less than half the size of selection in the two other years. In that year, selection for early flowering was not detectable without accounting for effects of leaf-out day. This suggests that accounting for differences in vegetative phenology can affect the ability to correctly estimate selection on flowering time. However, rather than overestimating the frequency of selection for early flowering, which we might have expected given the previous literature (e.g. Rathcke and Lacey 1985, Austen et al. 2017), neglecting variation in leaf-out time in our analyses would have led to underestimation of the strength of selection for early flowering.

In our study, phenotypic selection favoured short development times between leaf-out and first flowering day in two of three study years. We are unaware of any previous studies estimating selection on the time period between leaf-out and flowering. However, it has been hypothesised that rapid development to reproduction should be favoured since it decreases the likelihood of damage before reproduction (Williams 1966, Post et al. 2008). Still, the fitness effects of a short development time between leaf-out day and first flowering day are difficult to separate from the effects of early flowering, and a short development time might simply be favoured because it allows for early reproduction. It is also possible that selection for short development time is the result of that the optimal leaf-out time is close to the optimal timing of flowering initiation, and that the fitness benefits of a short development time reflect independent benefits of leafing out and flowering during a particular period.

While we found selection on the relative timing of leaf-out and first flowering day in terms of development time, we found no evidence of correlational selection on the two traits. That is, the benefits of a shorter development time were independent of the timing of leaf-out and flowering initiation. Estimates of correlational selection are overall rare (Kingsolver et al. 2001), and for plants, we are aware of only one study reporting significant correlational selection on combinations of phenological traits (flowering time and fruit maturation, Kelly 1992). To better understand how correlations among seasonal events affect the selection on a focal phenological trait, we therefore need studies estimating selection on combinations of seasonal events, as well as correlations and indirect selection for such events.

Our analyses of phenotypic selection provide important information about how selection can act on correlated life history traits. However, it is important to remember that our findings were based on female reproductive success and not on lifetime fitness, and it is possible that selection acting via male fitness differs from selection via female fitness. However, we consider it unlikely that including also male fitness would substantially alter our estimates of selection on phenological traits. Pollen is transferred between male and female parts of flowers that have a similar phenology in L. vernus, and it is likely that donating and recipient plants on average have a similar phenology. It is also true that trade-offs between current and future reproduction implies that some of the advantages of early flowering observed in this study might be offset by reduced fitness in subsequent years. Still, such costs of reproduction appear to be relatively modest in L. vernus, and the probability of flowering is higher in individuals that flowered in the previous year than individuals that were non-reproductive (Ehrlén and Van Groenendael 2001). Lastly, to predict evolutionary responses to observed selection, it is essential to know to what extent observed trait variation correspond to genetically based variation vs. plasticity. A previous study with $L$. vernusshowed that much of the variation in timing 
of flowering was plastic (Fogelström and Ehrlén 2019), suggesting that observed phenotypic selection might not correspond to genotypic selection and lead to evolutionary responses.

\section{Concluding remarks}

To obtain accurate estimates of phenotypic selection, it is essential to consider also selection via correlated traits (Lande and Arnold 1983). Yet, selection on the timing of life-cycle events is rarely estimated comprehensively enough to detect indirect selection via other phenological traits or correlational selection on trait combinations. Our results show that correlations between reproductive and vegetative phenology, in combination with contrasting selection on these two traits, can affect selection on flowering phenology, although the direction of selection on flowering time remained the same in our study. More generally, the results illustrate that selection on phenological traits can only be fully understood from the perspective of the seasonal development cycle. Yet, we still know little about the relationships among temporally correlated life history events, and the importance of these events for fitness. Insights into the extent to which selection on the timing of seasonal events is influenced by selection on temporally correlated events are also essential to understand long-term responses to anthropogenic climate change, as climate is likely to influence the phenotypic expression of sequential seasonal events as well as the optimal timing of these events (Li et al. 2016, Zohner et al. 2018, Mohan 2019).

\section{Data accessibility statement}

The datasets and code supporting this article are available athttps://fogelstrom.github.io/Elsa/data_files/Data_SimultaneousSelection.zipduring the review process, and will be uploaded to Dryad data repository once the paper is accepted for publication.

\section{Competing Interests}

The authors declare no conflict of interests.

\section{Authors' Contributions}

Elsa Fogelström : Conceptualization (supporting); Data curation (lead); Formal analysis (lead); Investigation (equal); Methodology (supporting); Writing-original draft (lead); Writing-review \& editing (lead). Giulia Zacchello : Investigation (equal). Johan Ehrlén : Conceptualization (lead); Data curation (supporting); Formal analysis (supporting); Funding acquisition (lead); Investigation (supporting); Methodology (lead); Resources (lead); Writing-original draft (supporting); Writing-review \& editing (supporting).

\section{Acknowledgments}

We thank Tove von Euler, Anna Herrström, Sara Jeschke-Sjoqvist, Annelie Jorgensen, Malin Konig, and Jessica Oremus for assistance in the field. We are grateful to Alicia Valdes and Ayco Tack who provided useful comments on previous versions of the manuscript. This work was supported by the Swedish Research Council (grant number 2019-04610 VR).

\section{References}

Aizen, M. A. 2003. Influences of animal pollination and seed dispersal on winter flowering in a temperate mistletoe. Ecology 84:2613-2627.

Augspurger, C. K. 2008. Early spring leaf out enhances growth and survival of saplings in a temperate deciduous forest. Oecologia 156:281-286.

Augspurger, C. K. 2013. Reconstructing patterns of temperature, phenology, and frost damage over 124 years: spring damage risk is increasing. Ecology 94:41-50.

Austen, E. J., L. Rowe, J. R. Stinchcombe, and J. R. Forrest. 2017. Explaining the apparent paradox of persistent selection for early flowering. New Phytologist 215:929-934. 
Bertin, R. I., and O. D. Sholes. 1993. Weather, pollination and the phenology of Geranium maculatum . American Midland Naturalist:52-66.

Brachi, B., C. Aime, C. Glorieux, J. Cuguen, and F. Roux. 2012. Adaptive value of phenological traits in stressful environments: predictions based on seed production and laboratory natural selection. PLoS ONE $7: 1-15$.

Brodie, E. D. 1992. Correlational selection for color pattern and antipredator behavior in the garter snake Thamnophis ordinoides . Evolution 46:1284-1298.

Canty, A., and B. Ripley. 2021. boot: Bootstrap R (S-Plus) Functions. R package version 1.3-27.

Dahlgren, J. P., H. v. Zeipel, and J. Ehrlen. 2007. Variation in vegetative and flowering phenology in a forest herb caused by environmental heterogeneity. American Journal of Botany 94:1570-1576.

Davison, A. C., and D. V. Hinkley. 1997. Bootstrap methods and their application. Cambridge University Press, Cambridge; New York, NY, USA.

De Lisle, S. P., and E. I. Svensson. 2017. On the standardization of fitness and traits in comparative studies of phenotypic selection. Evolution 71:2313-2326.

Diggle, P. K. 1999. Heteroblasty and the evolution of flowering phenologies. International Journal of Plant Sciences 160:S123-S134.

Ehrlen, J. 1992. Proximate limits to seed production in a herbaceous perennial legume, Lathyrus vernus . Ecology 73:1820-1831.

Ehrlen, J. 1996. Spatiotemporal variation in predispersal seed predation intensity. Oecologia 108:708-713.

Ehrlen, J. 2002. Assessing the lifetime consequences of plant-animal interactions for the perennial herb Lathyrus vernus (Fabaceae). Perspectives in Plant Ecology, Evolution and Systematics 5:145-163.

Ehrlen, J. 2015. Selection on flowering time in a life-cycle context. Oikos 124:92-101.

Ehrlen, J., and Z. Munzbergova. 2009. Timing of flowering: opposed selection on different fitness components and trait covariation. The American Naturalist 173:819-830.

Ehrlen, J., and A. Valdes. 2020. Climate drives among-year variation in natural selection on flowering time. Ecology Letters 23:653-662.

Elzinga, J. A., A. Atlan, A. Biere, L. Gigord, A. E. Weis, and G. Bernasconi. 2007. Time after time: flowering phenology and biotic interactions. Trends in Ecology \& Evolution 22:432-439.

Fogelstrom, E., and J. Ehrlen. 2019. Phenotypic but not genotypic selection for earlier flowering in a perennial herb. Journal of Ecology 00:1-10.

Fox, J., and S. Weisberg. 2019. An R companion to applied regression. Third edition. Sage, Thousand Oaks CA.

Harder, L. D., and S. D. Johnson. 2009. Darwin's beautiful contrivances: evolutionary and functional evidence for floral adaptation. New Phytologist 183:530-545.

Harrison, X. A., L. Donaldson, M. E. Correa-Cano, J. Evans, D. N. Fisher, C. E. D. Goodwin, B. S. Robinson, D. J. Hodgson, and R. Inger. 2018. A brief introduction to mixed effects modelling and multi-model inference in ecology. PeerJ 6.

Ida, T. Y., and G. Kudo. 2008. Timing of canopy closure influences carbon translocation and seed production of an understorey herb, Trillium apetalon (Trilliaceae). Annals of Botany 101:435-446.

Inouye, D. W. 2008. Effects of climate change on phenology, frost damage, and floral abundance of montane wildflowers. Ecology 89:353-362. 
Keenan, T. F., and A. D. Richardson. 2015. The timing of autumn senescence is affected by the timing of spring phenology: implications for predictive models. Global Change Biology 21:2634-2641.

Kelly, C. A. 1992. Spatial and temporal variation in selection on correlated life-history traits and plant size in Chamaecrista fasciculata . Evolution 46:1658-1673.

Kingsolver, J. G., H. E. Hoekstra, J. M. Hoekstra, D. Berrigan, S. N. Vignieri, C. E. Hill, A. Hoang, P. Gibert, and P. Beerli. 2001. The strength of phenotypic selection in natural populations. The American Naturalist 157:245-261.

Kingsolver, J. G., and D. W. Pfennig. 2004. Individual-level selection as a cause of Cope's rule of phyletic size increase. Evolution 58:1608-1612.

Lande, R., and S. J. Arnold. 1983. The measurement of selection on correlated characters. Evolution $37: 1210-1226$.

Li, X., L. Jiang, F. Meng, S. Wang, H. Niu, A. M. Iler, J. Duan, Z. Zhang, C. Luo, S. Cui, L. Zhang, Y. Li, Q. Wang, Y. Zhou, X. Bao, T. Dorji, Y. Li, J. Penuelas, M. Du, X. Zhao, L. Zhao, and G. Wang. 2016. Responses of sequential and hierarchical phenological events to warming and cooling in alpine meadows. Nature Communications 7:12489.

McKinney, A. M., and K. Goodell. 2010. Shading by invasive shrub reduces seed production and pollinator services in a native herb. Biological Invasions 12:2751-2763.

Mohan, J. E. 2019. Ecosystem Consequences of Soil Warming: Microbes, Vegetation, Fauna and Soil Biogeochemistry. Academic Press.

Munguia-Rosas, M. A., J. Ollerton, V. Parra-Tabla, and J. A. De-Nova. 2011. Meta-analysis of phenotypic selection on flowering phenology suggests that early flowering plants are favoured. Ecology Letters 14:511521.

O'Neil, P. 1997. Natural selection on genetically correlated phenological characters in Lythrum salicaria L. (Lythraceae). Evolution 51:267-274.

Phillips, P. C., and S. J. Arnold. 1989. Visualizing multivariate selection. Evolution 43:1209-1222.

Post, E. S., C. Pedersen, C. C. Wilmers, and M. C. Forchhammer. 2008. Phenological sequences reveal aggregate life history response to climatic warming. Ecology 89:363-370.

POWO. 2019. Plants of the World Online. Facilitated by the Royal Botanic Gardens, Kew. http://www.plantsoftheworldonline.org/.

R Core Team. 2021. R: a language and environment for statistical computing. R Foundation for Statistical Computing, Vienna, Austria.

Rathcke, B., and E. P. Lacey. 1985. Phenological patterns of terrestrial plants. Annual Review of Ecology \& Systematics 16:179-214.

Rausher, M. D. 1992. The measurement of selection on quantitative traits: biases due to environmental covariances between traits and fitness. Evolution 46:616-626.

Roy, B. A., S. Gusewell, and J. Harte. 2004. Response of plant pathogens and herbivores to a warming experiment. Ecology 85:2570-2581.

Sedlacek, J., J. A. Wheeler, A. J. Cortes, O. Bossdorf, G. Hoch, C. Lexer, S. Wipf, S. Karrenberg, M. van Kleunen, and C. Rixen. 2015. The response of the alpine dwarf shrub Salix herbacea to altered snowmelt timing: lessons from a multi-site transplant experiment. PLoS ONE 10:1-19.

Sola, A. J., and J. Ehrlen. 2007. Vegetative phenology constrains the onset of flowering in the perennial herb Lathyrus vernus. Journal of Ecology 95:208-216. 
Stinchcombe, J. R., A. F. Agrawal, P. A. Hohenlohe, S. J. Arnold, and M. W. Blows. 2008. Estimating nonlinear selection gradients using quadratic regression coefficients: double or nothing? Evolution 62:24352440 .

Williams, G. C. 1966. Adaptation and natural selection: a critique of some current evolutionary thought. Princeton university press.

Yao, Q., and S. A. Mehlenbacher. 2000. Heritability, variance components and correlation of morphological and phenological traits in hazelnut. Plant Breeding 119:369-381.

Zohner, C. M., L. Mo, and S. S. Renner. 2018. Global warming reduces leaf-out and flowering synchrony among individuals. Elife 7:e40214.

\section{Figure legends}

Fig. 1. Relationship between leaf-out day and first flowering day for Lathyrus vernus individuals in the years 2013, 2014 and $2015\left(\mathrm{n}_{2013}=198, \mathrm{n}_{2014}=207, \mathrm{n}_{2015}=207\right)$.

Fig. 2. Relationships between fitness and (a) leaf-out day, (b) first flowering day, and (c) the development time between leaf-out and first flowering day in Lathyrus vernus in the years 2013 (left panels), 2014 (midpanels) and 2015 (right panels). Each point represents the raw trait-fitness values for one individual. Dashed lines represent total selection predicted from multiple regression models with fitness (the number of intact seeds, relativized within years) as a response variable, and (a) leaf-out day, (b) first flowering day, or (c) the development time between leaf-out and first flowering day as the predictor variable, and plant size (aboveground volume) included as a covariate. Solid lines represent direct selection, predicted from multiple linear regression models as above, but with both leaf-out day and first flowering day included as predictors. All predictor variables were standardized within years to 0 mean and unit variance before analysis. Plant size was ln-transformed before standardization.

\section{Figures:}

Figure 1
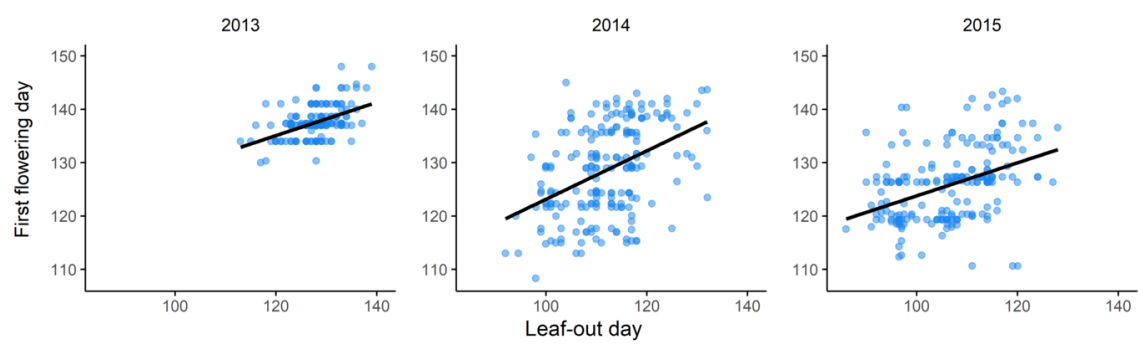

Figure 2 

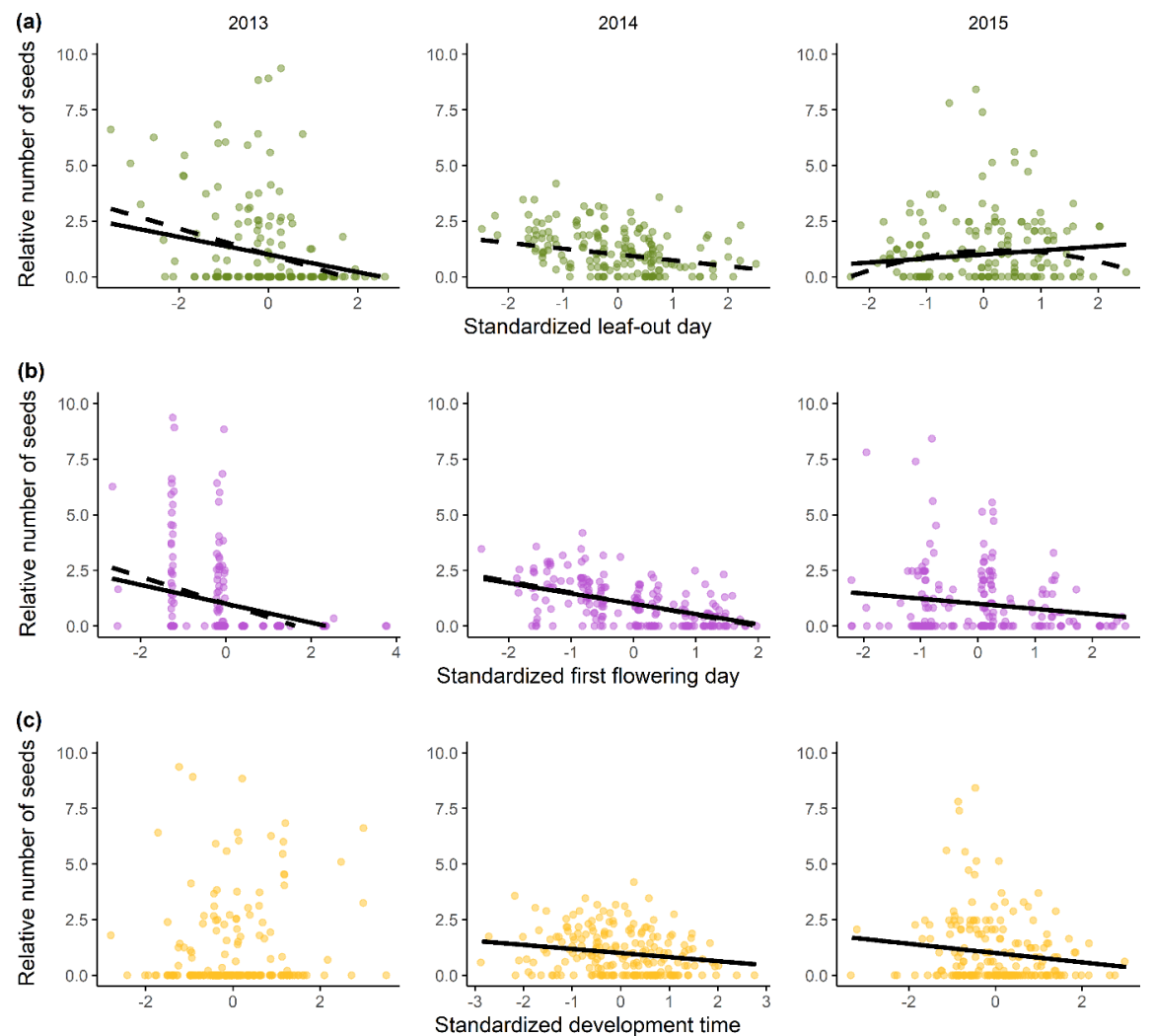

\section{Tables}

Table 1: Total selection on (a) leaf-out day and (b) first flowering day, and direct linear, nonlinear and correlational selection for both traits (c). Model estimates with $95 \%$ bias-corrected and accelerated (BCa) bootstrap intervals. Estimates with BCa intervals that do not overlap zero are inbold . Fitness (the response variable, the number of intact seeds) was relativized and leaf-out day, first flowering day, and plant size (above-ground volume) were standardized to 0 mean and unit standard deviation before analysis. Plant size was $\ln$-transformed before standardization. The nonlinear (quadratic) model estimates represent $\frac{1}{2}$ of the magnitude of nonlinear selection (Stinchcombe et al. 2008)

\begin{tabular}{llll}
\hline & 2013 & 2013 & 2013 \\
& Estimate & BCa interval & BCa inter \\
& & Lower & Upper \\
Total selection: Leaf-out day & Total selection: Leaf-out day & Total selection: Leaf-out day & Total selec \\
Leaf-out day & $\mathbf{- 0 . 5 8 4}$ & $\mathbf{- 0 . 8 1 6}$ & $\mathbf{- 0 . 3 4 7}$ \\
Plant size & $\mathbf{0 . 7 6 7}$ & $\mathbf{0 . 5 2 0}$ & $\mathbf{1 . 1 1 1}$ \\
Leaf-out day $^{2}$ & 0.096 & -0.073 & 0.227 \\
Total selection: First flowering day $^{-}$Total selection: First flowering day & Total selection: First flowering day & Total selec \\
First flowering day & $\mathbf{- 0 . 6 0 8}$ & $\mathbf{- 0 . 8 9 5}$ & $\mathbf{- 0 . 3 8 5}$ \\
Plant size & $\mathbf{0 . 6 9 1}$ & $\mathbf{0 . 4 3 5}$ & $\mathbf{1 . 0 2 4}$ \\
First flowering day & 0.109 & -0.070 & 0.276 \\
Direct selection & Direct selection & Direct selection & Direct sele \\
Leaf-out day & $\mathbf{- 0 . 3 9 6}$ & $\mathbf{- 0 . 6 5 9}$ & $\mathbf{- 0 . 1 2 7}$ \\
First flowering day & $\mathbf{- . 4 2 5}$ & $\mathbf{- 0 . 7 5 2}$ & $\mathbf{- 0 . 1 7 5}$
\end{tabular}


Plant size

Leaf-out day ${ }^{2}$

First flowering day ${ }^{2}$

Leaf-out day $\times$ First flowering day $\mathbf{0 . 6 8 5}$

0.107

0.055

0.024
0.433

$-0.051$

$-0.159$

$-0.322$
1.013

0.295

0.308

0.320 